\title{
A second-line escalating treatment strategy for children with severe chronic immune thrombocytopenia: A retrospective data from a single-center
}

\author{
lingling $\mathrm{FU}^{1}$, Jie $\mathrm{Ma}^{2}$, Hao $\mathrm{Gu}^{3}$, Jingyao $\mathrm{Ma}^{4}$, Yunyun $\mathrm{Wei}^{1}$, Zhenping $\mathrm{Chen}^{5}$, and Runhui \\ $\mathrm{Wu}^{2}$ \\ ${ }^{1}$ Beijing Children's Hospital, Capital Medical University, National Center for Children's \\ Health \\ ${ }^{2}$ Beijing Children's Hospital \\ ${ }^{3}$ Beijing Children's Hospital Capital Medical University \\ ${ }^{4}$ Beijing Children's Hospital Capital Medical University National Center of Children's \\ Health, China \\ ${ }^{5}$ Beijing Children's Hospital Capital Medical University
}

November 30, 2020

\begin{abstract}
Objective: To analyze the effect of a novel second-line escalating treatment strategy (high-dose dexamethasone (HDD), low-dose rituximab to eltrombopag) for children with severe chronic immune thrombocytopenia (SCITP). Materials and Methods: This study was a single-center, retrospective cohort study. The second-line escalating strategy included 3 steps: Step I (6 courses high-dose dexamethasone: HDD), Step II (HDD combined with low-dose rituximab), and Step III (eltrombopag). Results: A total of 30 cases (18 males and 12 females) were included; the median age was 8.83 (1.42-13.9) year-old, the duration time of ITP was 20.5 (12.0-96.0) months, and the platelet counts were 15 (3-29) ×109/L. After the median 14 (12-37) months' treatment, the remission rate was $36.7 \%$ (11/30), and the sustained response (SR) rate was $68.2 \%(15 / 22)$. In eltrombopag (step III) cases, $47.5 \%$ (8/17) maintained platelet [?]50x109/L, 37.5\% (3/8) dose tapering, and 25\% (2/8) were successfully discontinued from medication. The number of patients at $12 \mathrm{th}, 24 \mathrm{th}$, and $36 \mathrm{th}$ months was 30,7 , and 2 , with the total response (TR) and remission rates of $80 \%(36.7 \%), 57.1 \%(28.6 \%)$, and $50 \%(50 \%)$, respectively. The total relapse rate was $26.7 \%(8 / 30)$,three cases $(75 \%, 3 / 4)$ from Step II and 5 cases $(41.7 \%, 5 / 12)$ from Step III, none in Step I. Conclusion: The new second-line escalating strategy for children SCITP has an effective improving rate with $36.7 \%$ remission and $68.2 \%$ SR; $30 \%$ could benefit and retain stable response from HDD treatment. Combined treatment with eltrombopag can reduce the relapse rate of low-dose rituximab.
\end{abstract}

\section{Hosted file}

mian text.pdf available at https://authorea.com/users/379824/articles/496049-a-second-lineescalating-treatment-strategy-for-children-with-severe-chronic-immune-thrombocytopeniaa-retrospective-data-from-a-single-center 

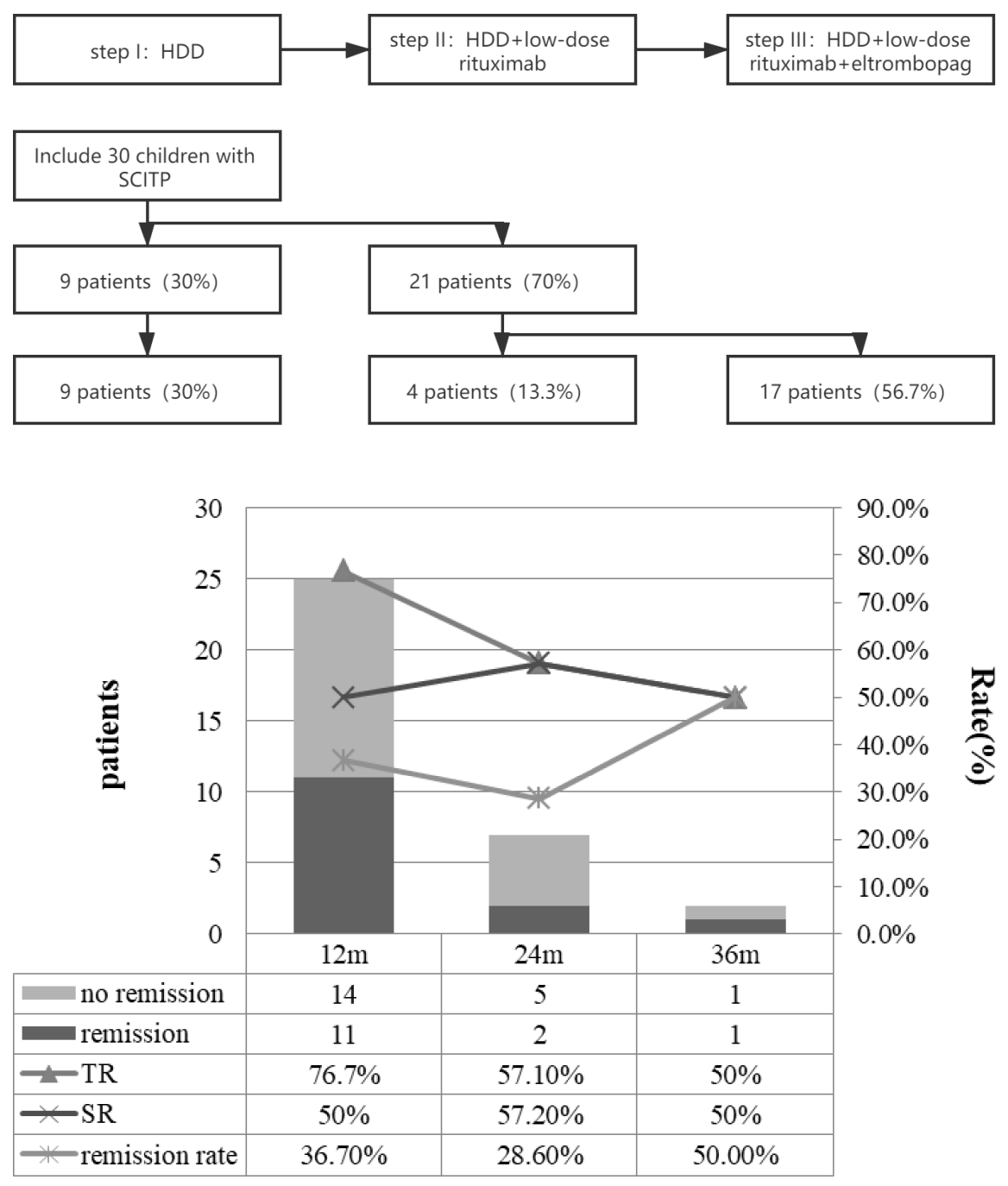

\section{Hosted file}

Table 1.pdf available at https://authorea.com/users/379824/articles/496049-a-second-lineescalating-treatment-strategy-for-children-with-severe-chronic-immune-thrombocytopeniaa-retrospective-data-from-a-single-center 